\title{
Reward and learning in honeybees: Analysis of an overshadowing effect
}

\author{
P. A. COUVILLON, ESTRELLITA T. MATEO, and M. E. BITTERMAN \\ University of Hawaii, Honolulu, Hawaii
}

\begin{abstract}
Previous experiments have shown that honeybees trained with colored targets baited with 5-versus 20- $\mu$ l drops of sucrose solution fail to develop a preference for the $20-\mu$ l color when the location of the drop on each target is marked by a white dot (dot-color overshadowing) but that discrimination is not impaired by dots when the targets differ in odor rather than in color. In Experiments 1-3, dot-color overshadowing failed to appear with differences in concentration rather than amount of sucrose (50\% vs. $20 \%$ or $0 \%$ ), but it did appear in Experiments 4 and 5 with a difference in probability of reward (consistent vs. partial). Experiment 6 showed no dot-odor overshadowing with a difference in probability of reward. The results are not generally predictable from the Rescorla-Wagner principle of shared associative strength, but point instead (in conjunction with those of earlier experiments) to competition for visual attention.
\end{abstract}

Foraging honeybees trained with successively presented targets of two different colors, one color always signaling a $5-\mu 1$ and the other a $20-\mu$ ldrop of $50 \%$ sucrose solution, quickly develop a preference for the $20-\mu \mathrm{l}$ color that is most simply explained in terms of a stronger association with sucrose (Buchanan \& Bitterman, 1988). Because 20- $\mu$ ldrops are detected somewhat more readily than 5- $\mu$ l drops (Walker, Lee, \& Bitterman, 1990), the stronger association might be due, not to the difference in amount of sucrose, but to closer contiguity between the perception of the $20-\mu \mathrm{l}$ color and the initial taste of sucrose, an interpretation supported by the fact that no preference develops when delay is equated by marking the location of the drop of sucrose at the center of each target with a salient white dot (Lee \& Bitterman, 1990). There is other evidence, however, to suggest that the preference found in work with undotted targets is indeed attributable to the difference in amount of sucrose, and that no preference develops when dotted targets are used because the colors are overshadowed by the dots, which themselves can be shown to acquire associative strength; for example, foragers that have been trained with a single dotted target containing a 5- $\mu$ l drop of sucrose solution prefer a dotted to an undotted target in an unrewarded choice test (Lee \& Bitterman, 1990).

The overshadowing interpretation is supported by the fact that when odors are substituted for colors in experiments of the same design, a preference for the $20-\mu$ lodor quickly develops whether or not dotted targets are used

This research was supported by Grant IBN-9308132 from the National Science Foundation. The participation of Estrellita T. Mateo was made possible by Minority Biomedical Research Support Grant GM08125-21 from the National Institutes of Health. Requests for reprints should be addressed to $M$. E. Bitterman, Békésy Laboratory of Neurobiology, 1993 East-West Road, Honolulu, HI 96822 (e-mail: jeffb@ahi.pbrc.hawaii.edu). to equate delay (Couvillon, Lee, \& Bitterman, 1991; Lee \& Bitterman, 1990); the reasonable implication of these results is that dots overshadow colors but not odors. The overshadowing interpretation is supported also by the fact that the $20-\mu \mathrm{l}$ preference appears even in work with colors when delay is equated, not by adding dots to the conventional flat targets, but by using targets of an inverted conical structure in which the animal is guided by the declining substrate to the location of reward at the base (Couvillon \& Bitterman, 1993).

Overshadowing commonly is thought to require a departure from the parsimonious independence assumptionthe assumption that the components of a compound stimulus gain or lose strength independently with reward or nonreward. To account for overshadowing, it has been proposed, for example, that the components of a compound compete with each other for associative strength (Rescorla \& Wagner, 1972) or for the attention assumed to be necessary for learning (Sutherland \& Mackintosh, 1971). Nevertheless, not all instances of overshadowing necessarily contradict the independence assumption. The prototypical Pavlovian effect can be explained in terms of generalization decrement. The fact that honeybees trained with a jasmine-scented orange target respond less when tested with an unscented orange target than do control subjects trained with the unscented orange target (Couvillon \& Bitterman, 1980) may be a reflection only of the greater dissimilarity of training and testing conditions for the experimental subjects than for the controls. Overshadowing in instrumental training sometimes can be attributed to different experience with the critical stimuli (Wagner, 1969). In choice experiments with honeybees (Couvillon \& Bitterman, 1989), subjects trained in a confounded color-odor problem (e.g., green-geraniol positive vs. blue-peppermint negative) learned less about the colors than did control subjects trained with color relevant and odor irrelevant, and less about the odors than 
did control subjects trained with odor relevant and color irrelevant (reciprocal overshadowing). As anticipated, however, the confounded problem was easier than the other two, which meant that the subjects trained in the confounded problem had relatively little unrewarded experience with the negative color and odor. The results were readily understandable, therefore, in terms of a simple theory incorporating the independence assumption with which it has been possible to account - quantitatively and with considerable precision-for the performance of honeybees in a wide range of color-odor discrimination problems (Couvillon \& Bitterman, 1991).

The overshadowing of colors by dots in training with colored targets containing different amounts of sucrose seems, however, to offer a more difficult challenge to the independence assumption. Although the number of exposures to each color under each condition was the same, there was a clear preference for the $20-\mu$ l color when the targets were undotted both in training and in the subsequent test, but no preference when the targets were dotted in training even if they were dotted in the test (an outcome that rules out an explanation in terms of generalization decrement). Our purpose in the present experiments was to inquire further into the conditions under which dot-color overshadowing occurs.

\section{EXPERIMENT 1}

In recent experiments analogous to those on amount of sucrose, color and odor preferences based on concentration of sucrose $(50 \%$ vs. $20 \%)$ were demonstrated with undotted targets (Loo \& Bitterman, 1992). Undotted targets were used, because, with drops of equal volume, there could be no confounding of concentration and delay. In the present experiment, with the same concentrations, the effects of training with dotted and undotted targets differing in color were compared. If the color-amount results were due simply to the overshadowing of the colors by the dots, analogous results could be expected with a difference in concentration substituted for the difference in amount.

\section{Method}

Subjects. The subjects were 32 honeybees (Apis mellifera), all experimentally naive, from our own hives situated near the laboratory. They were assigned in balanced order to four groups of 8 subjects each.

Procedure. The training situation was the same as that used in the color-amount experiments that showed the dot-color overshadowing effect (Lee \& Bitterman, 1990). It consisted of two immediately adjacent windows, each $58 \mathrm{~cm}$ wide and $58 \mathrm{~cm}$ high, separated by a thin $(2-\mathrm{cm})$ wooden partition around which the subject was required to fly from one window to the other. In the pretraining, experience with both windows was given. A single forager was selected at random from a group of foragers at a feeding station providing $10 \%-12 \%$ sucrose solution, carried to the laboratory, and set down at a $100-\mu \mathrm{l}$ drop of $50 \%$ sucrose solution on a pretraining target that was centered on the sill of one of the two windows, the left for half the subjects and the right for the rest. The subject was marked with a spot of colored lacquer as it fed to repletion, after which it was permitted to leave for the hive. Typi- cally, the subject would return to the laboratory after a few minutes, continuing to fly back and forth between the hive and the window as long as sucrose was available there. If the marked subject did not return after its first placement, it was carried again from the feeding station, where it usually could be found, to the pretraining target. When the subject did return to the first window, it was picked up after a few seconds and placed at a large drop of sucrose on another pretraining target that was centered on the sill of the alternative window, from which the subject left for the hive when replete. On subsequent visits, a pretraining target was presented twice at the second window and once again at the first. The pretraining ended after the subject had returned twice to each window of its own accord.

The targets were flat circles, $7.5 \mathrm{~cm}$ in diameter, of the same colored plastics used in previous color-amount experiments. In all, there were six sets of training targets. Those of one set were of orange plastic, and those of the second were of blue plastic. The targets of the third and fourth sets were like those of the first and second, except that at the center of each was a white dot, $4 \mathrm{~mm}$ in diameter. The targets of the fifth and sixth sets-dotted for subjects subsequently to be trained with dotted targets and undotted for subjects subsequently to be trained with undotted targets - were used only in the pretraining; each of them was half orange and half blue. The targets used were washed and exchanged for others in their sets after each visit to randomize extraneous stimuli.

Arriving from the hive on each training visit, a subject found either an orange or a blue target centered on the sill of one of the windows, the left window on half the visits and the right window on the rest, in quasi-random order. At the center of the target-on the white dot, if there was one-was a 5- $\mu$ l drop of sucrose solution. When the subject landed on the target and made contact with the reward, a second target-orange or blue, in quasi-random orderwas centered on the sill of the adjoining window, to which the subject would fly after taking the sucrose on the first target. Then the first target was removed, another new target was centered on the sill of the arrival window, and so forth, until the subject was replete and returned of its own accord to the hive. The training targets were dotted for two overshadowing groups, D-30 and D-60, and undotted for two control groups, ND-30 and ND-60. For half the subjects in each group, the concentration of sucrose was $50 \%$ on blue targets and $20 \%$ on orange targets, while the opposite was true of the remaining subjects in each group.

The procedure was such, of course, that the number of trials with each target could not be fixed exactly by the experimenter, since that depended on the behavior of the subjects - that is, on where in the series of trials given on each visit each subject broke off and returned to the hive. (The total intake of sucrose, averaging about $50 \mu$, varies to some extent from subject to subject and from visit to visit.) Nevertheless, a running record kept for each subject made it possible to come reasonably close to the planned frequencies of 30 trials with each color for groups D-30 and ND-30, and 60 trials with each color for groups D-60 and ND-60. The actual mean numbers of $50 \%$ trials, and $20 \%$ trials, and training visits for the subjects scheduled to receive 30 trials with each color were 32.4 , 32.0 , and 6.6 , respectively; and for the subjects scheduled to receive 60 trials with each color, $61.0,61.1$, and 10.7. The D and ND values were in each case essentially identical.

After its last training visit, each subject returned from the hive to find a pair of fresh targets, one orange and the other blue, both undotted, set $10 \mathrm{~cm}$ apart in a lateral arrangement on the sill of one of the windows, the left window for half the subjects in each group and the right window for the rest. For half the subjects in each group, the target of the $50 \%$ color was to the left of the other, and for the remaining subjects to the right, each target now containing a 5- $\mu$ l drop of tap water (unacceptable, and distinguishable from the sucrose solution only by taste). Upon encountering water on one of the targets, the subject would leave it, then return to it or go to the other, leave again, return again (sometimes only briefly, with 


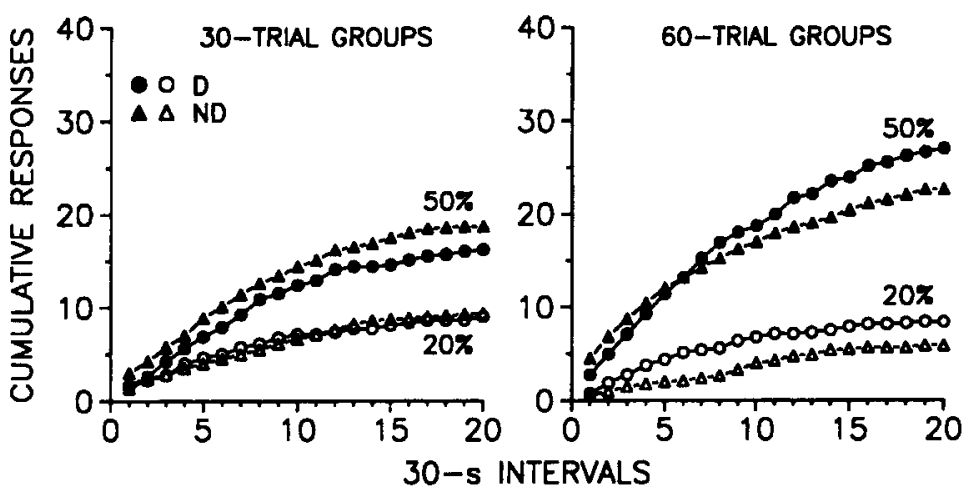

Figure 1. Performance of Groups D-30 and ND-30 (left) and of Groups D-60 and ND-60 (right) in unrewarded choice tests with the colors paired in training with $\mathbf{2 0 \%}$ or $\mathbf{5 0 \%}$ sucrose solution (Experiment 1). D, trained with dotted targets; ND, trained with undotted targets.

no attempt to drink), and so forth, the interval between successive responses increasing as the test continued. All actual contacts with each target, however brief, during a 10-min period were recorded by the experimenter, who pressed one of two hand-held switches that activated counters programmed to print stored frequencies at 30 -sec intervals.

\section{Results}

In the left-hand portion of Figure 1, the performance of Groups D-30 and ND-30 in the unrewarded choice test is plotted in terms of the mean cumulative number of responses to the $50 \%$ and $20 \%$ colors in successive 30 sec intervals. In the right-hand section of Figure 1, the test performance of groups D-60 and ND-60 is plotted in the same way. The curves suggest a preference for the $50 \%$ color that increases with the number of training trials but is unaffected by training condition (dots vs. no dots). Analysis of variance yields a significant stimulus $(50 \%$ vs. $20 \%$ ) effect $[F(1,28)=45.96, p<.0001]$ and a significant interaction of stimulus $\times$ amount of training $[F(1,28)=5.98, p=.0211]$, but neither a significant interaction of stimulus $\times$ training condition $(F<1)$ nor a significant three-way interaction of stimulus $\times$ amount of training $\times$ training condition $(F<1)$. In sum, no evidence of dot-color overshadowing appeared with a difference in concentration of sucrose instead of a difference in amount.

\section{EXPERIMENT 2}

In this experiment, we looked further for evidence of dot-color overshadowing with a simultaneous discrimination procedure that made it possible to examine the course of acquisition directly. On each trial, targets of two different colors were presented, one containing a drop of $50 \%$ sucrose solution and the alternative a drop of unacceptable tap water-here, that is, one of the colors was entirely unrewarded. Some subjects were trained with dotted targets and others with undotted targets, after which all subjects were tested with undotted targets.

\section{Method}

Subjects. The subjects were 24 honeybees, all experimentally naive, from our own hives situated near the laboratory. They were assigned in balanced order to two groups of 12 subjects each.

Procedure. The double-window situation was used, with recruitment and pretraining exactly the same as in Experiment 1 . The training also was very much the same, except that the discrimination was simultaneous rather than successive. Arriving from the hive on each training visit, the subject found, not a single target, but a pair of targets (one orange and one blue) set $10 \mathrm{~cm}$ apart on the sill of one of the windows. One of the two targets $(\mathrm{S}+)$, which was orange for half the subjects in each group and blue for the rest, contained a $10-\mu \mathrm{l}$ drop of $50 \%$ sucrose solution, while the other $(S-)$ contained a 10- $\mu$ d drop of tap water. The lateral arrangement of the targets varied over trials in quasi-random sequence. The initial choice made by the subject was recorded, with immediate correction of error permitted. As soon as the subject made contact with the sucrose on the $S+$ target, a second pair of targets was placed on the sill of the adjoining window, to which the subject would fly after finishing the sucrose on the target in the first window. Then a third trial was given in the arrival window, and so forth, until-typically after five trials-the subject was replete and left of its own accord for the hive. The training of each subject was continued for eight visits, both targets always dotted for Group D and always undotted for Group ND. The mean number of trials was 41.6 for Group D and 44.0 for Group ND. On the visit following the last training visit for each subject, there was an unrewarded 10-min choice test like that in Experiment 1, with orange and blue targets undotted for both groups, each target containing a $10-\mu 1$ drop of water.

\section{Results}

In Figure 2, the performance of the two groups is plotted in terms of the proportion of correct choice on each of the first 40 training trials. A somewhat unusual feature of the results is that the performance of both groups was somewhat poorer than chance at the outset, which is difficult to understand, because colors and other training variables were strictly balanced and because the animals showed neither a color nor a position preference. In any case, acquisition was very much the same in the two groups. Analysis of variance yields a significant effect of trials in blocks of four $[F(9,198)=23.56, p<.0001]$, 


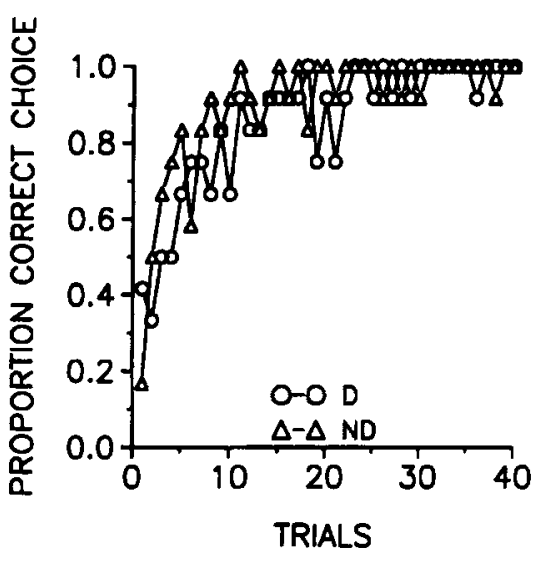

Figure 2. Performance of Groups D (dotted targets) and ND (undotted targets) in choice training with colors (Experiment 2).

but neither a significant group effect $(F<1)$, nor a significant group $\times$ trial-block interaction $(F<1)$.

In Figure 3, the performance in the unrewarded choice test with undotted targets is plotted in terms of the mean cumulative number of responses to the positive and negative colors in successive 30 -sec intervals, and here, too, the results for the two groups were very much the same. Analysis of variance yields a significant stimulus ( $\mathrm{S}+\mathrm{vs}$. $\mathrm{S}-)$ effect $[F(1,22)=110.01, p<.0001]$, but neither a significant group effect $(F<1)$ nor a significant group $X$ stimulus interaction $(F<1)$. Again the results give no indication of dot-color overshadowing.

\section{EXPERIMENT 3}

Here, as in Experiment 2, we looked for dot-color overshadowing in discriminative training with sucrose versus water, but under conditions that might be more likely to produce the effect: In the hope of minimizing the perceived difference between them, the colors were presented, not simultaneously, but successively (as they were

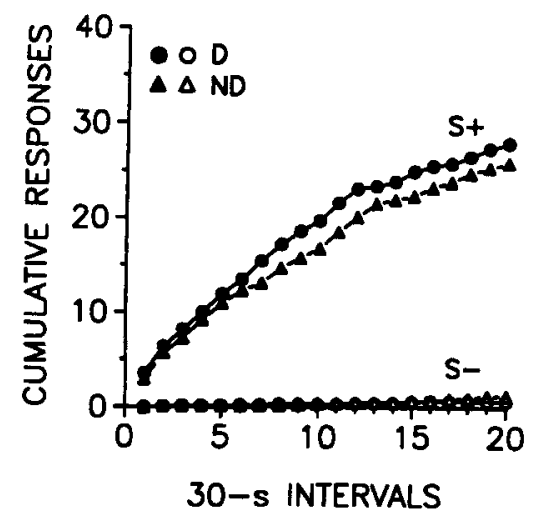

Figure 3. Performance of Groups D (trained with dotted targets) and ND (trained with undotted targets) in unrewarded choice tests with the $S+$ and $S-$ training colors (Experiment 2). in all the prior experiments that showed dot-color overshadowing). Another feature of the present experiment is that the number of training trials was varied on the chance of an overshadowing effect that would wash out in the course of prolonged training (Bellingham \& Gillette, 1981).

\begin{abstract}
Method
Subjects. The subjects were 32 honeybees, all experimentally naive, from our own hives situated near the laboratory. They were assigned in balanced order to four groups of 8 subjects each.

Procedure. Each subject was recruited as in the preceding experiments, but now to the sill of a single window, where it was placed at a large drop of $50 \%$ sucrose solution on a split orangeblue target, marked, and permitted to feed to repletion. The first return was to another such target, and the second was to a distinctive gray petri dish, $5.5 \mathrm{~cm}$ in diameter, with feeding to repletion on each. The training targets were the same dotted and undotted orange and blue plastic circles $(7.5 \mathrm{~cm}$ in diameter) used in the preceding experiments, each color positive for half the subjects in each group. When the subject returned from the hive on each training visit, it found a target of the negative color $(\mathrm{S}-$ ) containing a $10-\mu$ drop of water that remained for $30 . \mathrm{sec}$ on each of the first two visits, $60 \mathrm{sec}$ on each of the next two visits, and $120 \mathrm{sec}$ on each succeeding visit. All contacts with the target during that period were recorded, after which it was replaced with a target of the positive color $(S+)$ containing a $10-\mu 1$ drop of $50 \%$ sucrose solution. When the subject finished the sucrose and flew up, the positive target was replaced with the gray petri dish containing a large drop of $50 \%$ sucrose solution from which the subject fed to repletion and returned to the hive. That is, on each trial there was a period of exposure to an $\mathrm{S}-$ target that was followed by a small reward on an $\mathrm{S}+$ target and then by feeding to repletion on a target that was markedly different from both. Group $6 \mathrm{D}$ had 6 training visits (trials) with dotted targets; Group 12D, 12 training visits with dotted targets; Group 6ND, 6 training visits with undotted targets; and Group $12 \mathrm{ND}, 12$ training visits with undotted targets. On the visit following the last training visit for each subject, there was a 10 -min choice test with undotted orange and blue targets containing $10-\mu$ l drops of water.
\end{abstract}

\section{Results}

The measure of performance in training was the rate of responding on each visit, which declined over visits from a high of 8.5 responses per minute to 3.6 on the 6 th visit and (for the 12-visit groups) to 2.2 on the 12 th visit. The decline was significant both for the 6-visit groups $[F(5,70)=6.97, p<.0001]$ and for the 12-visit groups $[F(11,154)=10.48, p<.0001]$. It is impossible, of course, to say to what extent the decline reflected learning about the negative color and to what extent learning not to persist after an encounter with water. There was a small but statistically significant tendency for Group 12D to respond more than Group $12 \mathrm{ND}[F(1,14)=4.78, p=$ .0463 ], but the 6-visit groups did not differ significantly from each other $(F<1)$, nor was there a significant group $\times$ visit interaction with either amount of training $(F<1)$.

In the left-hand portion of Figure 4, the performance of the 6-visit groups in the unrewarded choice test is plotted in terms of the mean cumulative number of responses to the undotted positive and negative colors in successive $30-\mathrm{sec}$ intervals. In the right-hand portion of 


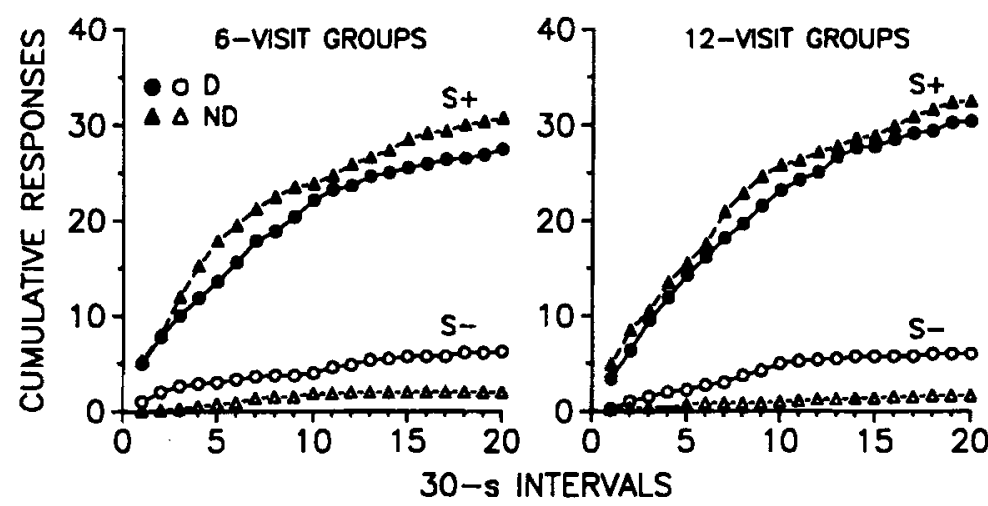

Figure 4. Performance of Groups 6D and 6ND (left) and of Groups 12D and 12ND (right) in unrewarded choice tests with the $S+$ and $S$ - training colors (Experiment 3). D, trained with dotted targets; ND, trained with undofted targets.

Figure 4, the test performance of the 12-visit groups is plotted in the same way. Analysis of variance yields a significant stimulus (S+ vs. $S-$ ) effect $[F(1,28)=131.66$, $p<.0001]$, but no significant treatment effect $(F<1)$ and no significant interaction either of stimulus $\times$ training condition (dots vs. no dots) $[F(1,28)=2.33, p>.05]$, or of stimulus $X$ amount of training $(F<1)$, or of stimulus $\times$ training condition $\times$ amount of training $(F<1)$. Again in this experiment, there is no reliable indication of overshadowing.

\section{EXPERIMENT 4}

Having failed in what were in effect three experiments on sucrose concentration ( $50 \%$ vs. $20 \%$ in the first and $50 \%$ versus $0 \%$ in the second and third) to find the dotcolor overshadowing discovered in prior experiments on amount of reward ( $5 \mu \mathrm{l}$ vs. $20 \mu \mathrm{l}$ of a $50 \%$ solution), we turned here to probability of reward, a third reward parameter of demonstrated importance in the learning of honeybees (Fischer, Couvillon, \& Bitterman, 1993; Ishida, Couvillon, \& Bitterman, 1992). The targets of two different colors-dotted for one group of animals and undotted for a second group - were presented successively in the two-window situation, with response to one color always rewarded and to the other rewarded only on half the training trials, after which there was a choice test with undotted targets. Negative results would suggest that the overshadowing effect is unique to differential conditioning based on amount of reward and understandable perhaps in terms of the way in which differences in amount of reward are detected, which is now far from clear (Batson, Hoban, \& Bitterman, 1992).

\section{Method}

Subjects. The subjects were 32 honeybees, all experimentally naive, from our own hives situated near the laboratory. They were assigned in balanced order to two groups of 16 subjects each.

Procedure. The subjects were recruited to the double-window situation and pretrained with split orange-blue targets exactly as in Experiment 1 . The training also was very much the same, except that here the attempt was to establish a color preference based on probability of reward rather than on concentration of sucrose. Targets of one color (the consistently rewarded color, which was orange for half the subjects and blue for the rest) always contained a $10-\mu l$ drop of $50 \%$ sucrose solution. Targets of the other color (the partially rewarded color) contained a $10-\mu$ l drop of $50 \%$ sucrose solution on half the trials and a $10-\mu$ l drop of water on the rest. When a subject tasted a drop of water, it would fly up from the target, which would be removed and then replaced when the animal went to the adjacent window. Trials with the two colors were scheduled in quasi-random sequence, as were rewarded and nonrewarded trials with the partially rewarded color. The plan was to have 24 trials with the consistently rewarded color, and 12 rewarded and 12 nonrewarded trials with the partially rewarded color. The actual (mean) numbers for Group D (trained with dotted targets) were $25.4,12.6$, and 12.9 , respectively, in 8.3 training visits; the corresponding means for Group ND (trained with undotted targets) were $23.6,12.2$, and 12.4 in 8.2 training visits. On the visit following the last training visit for each subject, there was a 10-min choice test with undotted orange and blue targets, each containing a $10-\mu$ ldrop of water.

\section{Results}

In Figure 5, the performance of the two groups in the unrewarded choice test is plotted in terms of the mean cumulative number of responses to the consistently and

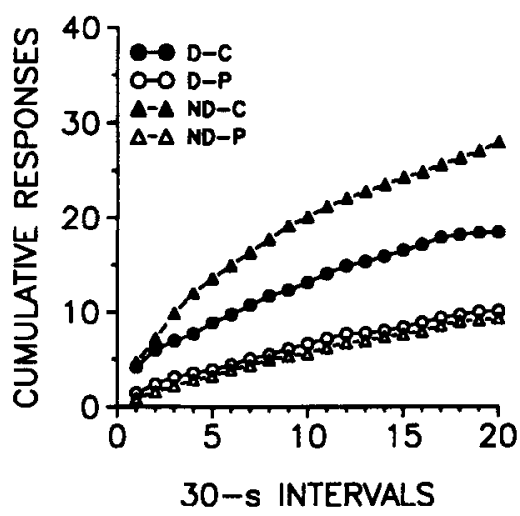

Figure 5. Performance of Groups D (trained with dotted targets) and ND (trained with undotted targets) in unrewarded choice tests with colors consistently $(C)$ and partially $(P)$ rewarded in training (Experiment 4). 
partially rewarded colors in successive 30 -sec intervals. The curves show a smaller preference for the consistently rewarded color in Group D than in Group ND. Analysis of variance yields a significant stimulus (consistent vs. partial) effect $[F(1,30)=44.92, p<.0001]$, without a significant group effect $[F(1,30)=2.99, p>$ $.05]$, but a significant group $\times$ stimulus interaction $[F(1,30)=6.58, p=.0155]$. That the dots did not entirely prevent discrimination of the colors as they did in the amount experiments (Lee \& Bitterman, 1990) is shown by a separate analysis of the test performance of Group D, which yields a significant stimulus effect $[F(1,15)=55.22, p<.0001]$. There was overshadowing, but it was not complete.

\section{EXPERIMENT 5}

Our purpose in this experiment was to try to confirm under a substantially different set of conditions the finding of Experiment 4 that dots may impair the discrimination of colors rewarded with different probabilities. The procedure employed here was like that of Experiment 3 , in which only a single trial with each color was given on each visit. Another change from the conditions of Experiment 4 designed to estimate the generality of its results was that an equated-reinforcements rather than an equated-trials design was employed - the same number of rewarded responses to each color and fewer trials with the consistently rewarded color, rather than the same number of trials with each color and fewer rewarded responses to the partially rewarded color. (It may be well to note that number of trials and number of rewards cannot both be equated in a comparison of partial and consistent reward.)

\section{Method}

Subjects. The subjects were 32 honeybees, all experimentally naive, from our own hives situated near the laboratory. They were assigned in balanced order to two groups of 16 subjects each.

Procedure. Each subject was recruited to a single window and pretrained exactly as in Experiment 3 . The 16 training visits were of four different kinds, and four of each were scheduled in quasirandom sequence. On visits of one kind, a target of the consistently rewarded color (orange for half the subjects in each group, and blue for the rest) was presented with a $10-\mu 1$ drop of $50 \%$ sucrose solution. The target then was replaced with a gray petri dish containing a large drop of $50 \%$ sucrose solution from which the subject fed to repletion. Visits of a second kind were like those of the first kind, except that the target was of the partially rewarded color. On visits of the third and fourth kinds, the subject found a target of the partially rewarded color containing a $10-\mu$ ld drop of water that remained for $60 \mathrm{sec}$ on the first such visit, $90 \mathrm{sec}$ on the next, and $120 \mathrm{sec}$ on the rest, during which time all contacts with the target were recorded. Then the target was replaced with a target containing a $10-\mu$ l drop of sucrose that was of the consistently rewarded color on visits of the third kind and of the partially rewarded color on visits of the fourth kind, each trial ending with feeding to repletion on a gray dish. In sum, with the consistently rewarded color designated as $\mathrm{C}$ and the partially rewarded color as $\mathrm{P}$, the training comprised four $\mathrm{C}+$ visits, four $\mathrm{P}+$ visits, four $\mathrm{P}-$ to $\mathrm{C}+$ visits, and four $\mathrm{P}-$ to $\mathrm{P}+$ visits (in balanced order). The only difference in the treatment of the two groups of subjects was

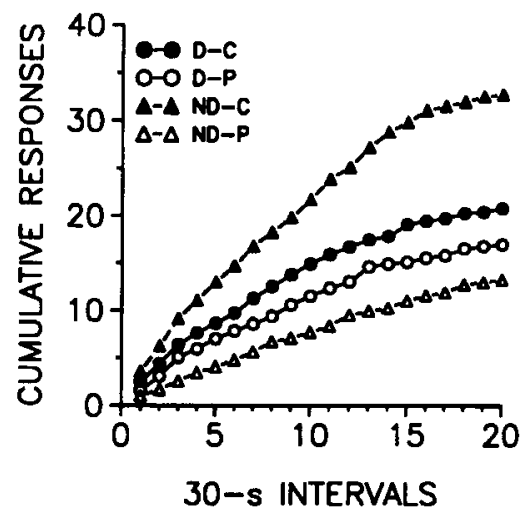

Figure 6. Performance of Groups D (trained with dotted targets) and ND (trained with undotted targets) in unrewarded choice tests with colors consistently $(C)$ and partially $(P)$ rewarded in training (Experiment 5).

that the orange and blue targets were dotted for Group D and undotted for Group ND. On the visit following the 16th training visit, there was an unrewarded 10 -min choice test with undotted orange and blue targets as in the preceding experiments.

\section{Results}

There was a significant decline in the rate of responding to the partially rewarded color (from about 7 to about 4 per minute) over the eight visits that began with unrewarded presentation of that color $[F(7,210)=9.45, p<$ $.0001]$, but with neither a group effect $(F<1)$ nor a group $\times$ stimulus interaction $(F<1)$. In the choice test, evidence of dot-color overshadowing appeared again, as is shown by Figure 6, in which the performance of the two groups is plotted in terms of the mean cumulative number of responses to the consistently and partially rewarded colors in successive 30-sec intervals. Analysis of variance yields a significant stimulus (consistent vs. partial) effect $[F(1,30)=14.33, p<.0001]$, without a significant group effect $[F(1,30)=2.32, p>.05]$, but a significant group $\times$ stimulus interaction $[F(1,30)=6.49$, $p=.0162]$. A separate analysis of the test performance of Group D does not yield a significant stimulus effect $(F<1)$; statistically, the overshadowing was complete.

\section{EXPERIMENT 6}

Overshadowing by dots has been found in discrimination experiments with colored targets but not with scented targets containing different amounts of reward (Lee \& Bitterman, 1990). In Experiments 4 and 5, dotcolor overshadowing was found with variation in probability of reward, and in the present experiment, patterned exactly after Experiment 5, a like effect was sought with odor. Negative results would support the hypothesis that colors and odors are differently affected by the dots.

\section{Method}

Subjects. The subjects were 32 honeybees, all experimentally naive, from our own hives situated near the laboratory. They were assigned to two groups of 16 subjects each. 


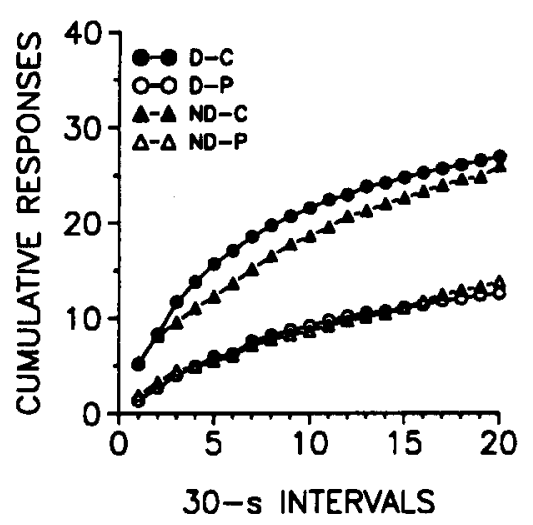

Figure 7. Performance of Groups D (trained with dotted targets) and ND (trained with undotted targets) in unrewarded choice tests with odors consistently $(C)$ and partially $(P)$ rewarded in training (Experiment 6).

Procedure. This experiment differed from Experiment 5 only in that scented gray targets were substituted for the colored ones. Here the targets were covered plastic petri dishes, $5.5 \mathrm{~cm}$ in diameter. Drilled in the cover of each dish, $6 \mathrm{~mm}$ from its outer circumference, was a circle of 16 equally spaced holes, $3 \mathrm{~mm}$ in diameter. The dishes contained pieces of cotton batting that could be impregnated as required with the scent of peppermint or with geraniol or (for purposes of pretraining only) with both. In all, there were six sets of such targets - scented in the three different ways and with or without a white dot, $4 \mathrm{~mm}$ in diameter, centered on the cover. To randomize extraneous stimuli, each target used on any visit was drawn from a large set of identical targets to which it was returned after the washing of its cover at the end of the visit. Distinctive dishes of a seventh set, unscented, were used to present the large drops of sucrose solution from which there was the feeding to repletion with which each visit ended.

\section{Results}

There was a significant decline in rate of responding to the partially rewarded odor (from about 6 to about 4 per minute) over the eight visits that began with unrewarded presentation of that odor $[F(7,210)=9.44, p \leqslant$ $<.0001]$, with neither a group effect $(F<1)$ nor a group $\times$ stimulus interaction $(F<1)$. In Figure 7 , the performance of the two groups in the choice test is plotted in terms of the mean cumulative number of responses to the consistently and partially rewarded odors in successive $30-\mathrm{sec}$ intervals. Analysis of variance yields a significant stimulus (consistent vs. partial) effect $[F(1,30)=74.20$, $p<.0001]$, a significant change in responding over 2.5min blocks $[F(3,90)=65.04, p<.0001]$, and a significant stimulus $\times$ block interaction $[F(3,90)=37.66, p<$ $.0001]$, but neither a significant group effect $(F<1)$ nor a significant group $\times$ stimulus interaction $(F<1)$.

\section{DISCUSSION}

In experiments with targets containing different amounts of reward ( 5 vs. $20 \mu \mathrm{l}$ of sucrose solution), Lee and Bitterman (1990) found overshadowing of colors, but not of odors, by dots that marked the location of re- ward, and parallel results-dot-color but not dot-odor overshadowing - were obtained here for different probabilities of reward (consistent vs. partial). It might be suspected that the odors employed were more salient or discriminable than the colors, but there is no support for that interpretation. In both the amount and the probability experiments, discrimination of the colors by animals trained with undotted targets was better if anything than discrimination of the odors; compare, for example, the ND-C and ND-P curves for color in Figure 6 and for odor in Figure 7.

Another interpretation worth considering is that independence is more likely in intermodal compounds (compounds whose components are of different modalities, such as color and odor) than in intramodal compounds (compounds whose components are of the same modality, such as dot and color). Much the same proposal has been made by Kehoe, Horne, Horne, and Macrae (1994) on the basis of discrepant results for tone-noise versus tone-light and noise-light compounds obtained in classical conditioning experiments with rabbits. On the assumption that the position of a target is given visually-either by reference to the surround, or by location in the visual field as determined by bodily orientation (Huber et al., 1994; Wehner, 1981) - it is possible to point to other experiments with honeybees whose results for intramodal compounds (specifically, color-position compounds) are at odds with the independence assumption, while those for intermodal (color-odor) compounds are not.

Couvillon, Klosterhalfen, and Bitterman (1983) found reciprocal overshadowing of color and position in an earlier experiment of the same design as the color-odor overshadowing experiment by Couvillon and Bitterman (1989) that has already been described. Foragers trained in a confounded color-position problem (e.g., greenright positive vs. blue-left negative) learned less about color than did control subjects trained with color relevant and position irrelevant, and less about position than did control subjects trained with position relevant and color irrelevant. Unlike the color-odor results, the colorposition results cannot be accounted for in terms of differential experience with the negative stimuli, because the confounded problem proved to be no easier than either the color-relevant problem or the position-relevant problem. Summation in intramodal compounds failed to appear in the experiments of Kehoe et al. (1994) with rabbits, and still more recently also in autoshaping experiments with pigeons by Rescorla and Coldwell (1995).

In a still earlier experiment, Klosterhalfen, Fischer, and Bitterman (1978) found dimensional transfer-a vertebrate phenomenon long taken as evidence of selective attention (Sutherland \& Mackintosh, 1971)-in color-position problems although not in color-odor problems: Foragers trained first in a color-odor problem with color or odor relevant, and then in a second such problem with new colors and odors, performed no better in the second problem when the relevant dimension was the same as in the first problem than when it was differ- 
ent; but after training in a color-position problem with color or position relevant, performance in a second problem with new colors and positions was better when the relevant dimension was the same as in the first problem than when it was different. Here again an explanation of the color-position results in terms of competition for visual attention is suggested.

Yet another interpretation worth considering is that the discrepant results for color-odor as compared with color-position compounds are due, not to the intermodality of color-odor compounds, but to some special way in which position is processed. It may be important, for example, that color and odor are integral properties of a target while position is not; a distinctive manner of choice in position-relevant problems was, in fact, reported in both papers on color-position training. The question could be decided by a dimensional transfer experiment with odor and position, which should give positive results if position qua position is critical, but not if intramodality is critical. Support for the interpretation in terms of intramodality is provided by recent work on blocking. Color-odor experiments with free-flying foragers have failed to turn up evidence of blocking (Funayama, Couvillon, \& Bitterman, 1995), but blocking has been found in experiments on proboscis-extension conditioning in harnessed foragers exposed to binary odor mixtures that were treated as odor-odor compounds (Smith \& Cobey, 1994).

For the moment, however, the concept of competition for visual attention does make it possible to understand why there should be dot-color overshadowing but not dot-odor overshadowing, and perhaps also why there should be dot-color overshadowing with different amounts and probabilities of sucrose but not with different concentrations. In the training with two amounts of reward, selective attention to the salient dots would have been maintained and even strengthened because of their close and consistent relation to the initial taste of $50 \%$ sucrose; the many versions of traditional attention theory have in common the assumption that the strength of attention to some feature of a stimulus is correlated with the probability of reward for response to it (Sutherland \& Mackintosh, 1971). Although the dots did not predict amount of sucrose, it is easy to think that concentration is a more immediate and salient property of reward than amount, which most likely is discriminated only as ingestion continues. In training with two concentrations, attention to the dots would have given way to attention to the colors, because the taste of sucrose was predicted by the colors and not by the dots. In training with two probabilities, the dots were at least partial predictors of sucrose-paired with it twice as often as with water in one case (Experiment 5) and three times as often in the other (Experiment 4) - and that experience, together with their greater salience, may have been sufficient to give them an advantage over the colors. From this viewpoint, the overshadowing effect would be expected to diminish with further reduction of the dot-sucrose correlation; it might be instructive, for example, to train animals with one color rewarded on $75 \%$ of trials and the other on $25 \%$ of trials.

The principle of shared associative strength (Rescorla \& Wagner, 1972) provides no immediate explanation either of dimensional transfer or of the discrepant results obtained in analogous experiments with different modalities. Nevertheless, it may be instructive to ask how well dot-color overshadowing alone can be accounted for in terms of that principle, an attractive feature of which is that it is expressed in equational form. It will not do, of course, simply to try to simulate the experiments with some arbitrarily chosen values of the several parameters (cf. Pearce \& Redhead, 1993, on the effect of a common irrelevant stimulus on positive and negative patterning in pigeons), because different sets of parameter values may well give results that are qualitatively quite different. What is required is factorial variation of parameter values over a wide range, which was done here for three problems.

In simulations of training with two amounts of reward (20 and $5 \mu \mathrm{l}$ ), there were 100 trials each with $\mathrm{AX}_{20}$ and $\mathrm{BX}_{5}$ for an overshadowing group and with $\mathrm{A}_{20}$ and $\mathrm{B}_{5}$ for a control group (the colors represented by $\mathrm{A}$ and $\mathrm{B}$, the dots by $\mathrm{X}$ ). The associative strength supported by the larger reward, $\lambda_{20}$, was taken as $1 ; \lambda_{5}$ was varied from .1 to .9 in steps of .1; $\alpha_{\mathrm{C}}$, the common salience of the colors was varied from. 1 to 1 in steps of $.1 ; \alpha_{X}$, the salience of $X$ was varied from .1 to 1 in steps of .1; UB (the incremental $\beta$ ) was varied from .02 to .3 in steps of .02 , and so also (independently) was $D \beta$ (the decremental $\beta$ ). Of the 202,500 simulations, $43 \%$ showed at least some overshadowing as reflected by an asymptotic difference in the associative strengths of $A$ and $B\left(V_{A}-V_{B}\right)$ larger for the control group than for the overshadowing group. The largest effect was .53. In the sets of parameter values yielding more than very small overshadowing effects, UB ranges from .1 -.3 , but they all have in common that $\mathrm{D} \beta$ is very small (always .02), $\alpha_{\mathrm{X}}$ is large relative to $\alpha_{\mathrm{C}}$, and $\lambda_{5}$ is very small (.1 or .2)-perhaps unrealistically small, given the substantial reinforcing properties of $5-\mu 1$ drops of $50 \%$ sucrose solution that have been demonstrated repeatedly (see, e.g., Couvillon et al., 1991; Lee \& Bitterman, 1990). It should be noted that the simulation procedure is exactly the same for two different concentrations as for two different amounts, and the results for amount apply directly to concentration.

As might be anticipated, results similar to those for two amounts (or concentrations) were obtained in simulations of training with A rewarded $(10 \mu \mathrm{l})$ and B entirely unrewarded (as in Experiments 2 and 3)-100 trials each with $\mathrm{AX}_{10}$ and $\mathrm{BX}-$ for an overshadowing group and with $A_{10}$ and $B-$ for a control group. The simulation procedure was in general the same as before, except that the asymptotic strength of $B$ was fixed at zero, with $\lambda_{10}$ varied from .1-1 in steps of .1. Of 225,000 simulations, $44 \%$ showed at least some overshadowing. The largest effect was .59. In the sets of parameter values that yielded more 
than very small effects, $\lambda_{10}$ was relatively large (.5-1), and the other values were much the same as in the twoamount case-D $B$ very small (always .02), and $\alpha_{X}$ large relative to $\alpha_{\mathrm{C}}$. The theory suggests that training with $\mathrm{A}$ rewarded and $B$ entirely unrewarded is a special case of training with two different amounts or concentrations.

Different results were obtained in simulations of training with two probabilities of reward -100 trials each with $\mathrm{AX}_{10}, \mathrm{BX}_{10}$, and $\mathrm{BX}$ - for an overshadowing group and with $\mathrm{A}_{10}, \mathrm{~B}_{10}$, and $\mathrm{B}-$ for a control group. Of 225,000 simulations, $34 \%$ yielded overshadowing effects, the magnitude of only 52 of them exceeding .03, with .042 the largest. The 52 sets of parameter values differ from those yielding substantial overshadowing effects with $B$ paired consistently with small reward or no reward in that both $U ß$ and $D ß$ are large (about .3), and that both $\alpha_{X}$ and $\alpha_{C}$ are large (about 1).

It seems, then, that the principle of shared associative strength does not help to understand the dot-color results. Although the principle suggests overshadowing when $A$ and $B$ provide either different amounts or different concentrations of sucrose, the results show overshadowing with a difference in amount but not with a difference in concentration. The principle also suggests that there should be little or no overshadowing when A is consistently rewarded and B only partially rewarded, but the results shown substantial overshadowing. There is, of course, some evidence recently reviewed by Miller, Barnet, and Grahame (in press) that overshadowing and blocking in vertebrates are performance effects rather than acquisition effects. If that should prove true of dotcolor overshadowing in future work with honeybees, it still might be reasonable to look for an explanation in terms of traditional attention theory, which postulates that the stimuli attended to and the associative properties of those stimuli are determined by separate processes.

\section{REFERENCES}

Batson, J. D., Hoban, J. S., \& Bitterman, M. E. (1992). Simultaneous conditioning in honeybees (Apis mellifera). Journal of Comparative Psychology, 106, 114-119.

Bellingham, W. P., \& Gillette, K. (1981). Attenuation of overshadowing as a function of nondifferential compound conditioning trials. Bulletin of the Psychonomic Society, 18, 218-220.

Buchanan, G. M., \& Bitterman, M. E. (1988). Learning in honeybees as a function of amount and frequency of reward. Animal Learning \& Behavior, 16, 247-255.

Couvillon, P. A., \& Bitterman, M. E. (1980). Some phenomena of associative learning in honeybees. Journal of Comparative \& Physiological Psychology, 94, 878-885.

Couvillon, P. A., \& Bitterman, M. E. (1989). Reciprocal overshadowing in the discrimination of color-odor compounds by honeybees: Further tests of a continuity model. Animal Learning \& Behavior, 17, 213-222.

Couvillon, P. A., \& Bitterman, M. E. (1991). How honeybees make choices. In J. L. Goodman \& R. C. Fischer (Eds.), The behaviour and physiology of bees (pp. 116-130). Wallingford, U.K.: CAB International.
Couvillon, P. A., \& Bitterman, M. E. (1993). Learning in honeybees as a function of amount of reward: Further experiments with color. Animal Learning \& Behavior, 21, 23-28.

Couvillon, P. A., Klosterhalfen, S., \& Bitterman, M. E. (1983). Analysis of overshadowing in honeybees. Journal of Comparative Psychology, 97, 154-166.

Couvillon, P. A., Lee, Y., \& Bitterman, M. E. (1991). Learning in honeybees as a function of amount of reward: Rejection of the equalasymptote assumption. Animal Learning \& Behavior, 19, 381-387.

Fischer, M. E., Couvillon, P. A., \& Bitterman, M. E. (1993). Choice in honeybees as a function of the probability of reward. Animal Learning \& Behavior, 21, 187-195.

Funayama, E. S., Couvillon, P. A., \& Bitterman, M. E. (1995). Compound conditioning in honeybees: Blocking tests of the independence assumption. Animal Learning \& Behavior, 23, 429-437.

Huber, B., Couvillon, P. A., \& Bitterman, M. E. (1994). Place and position learning in honeybees (Apis mellifera). Journal of Comparative Psychology, 108, 213-219.

Ishida, M., Couvillon, P. A., \& Bitterman, M. E. (1992). Acquisition and extinction of a shuttling response in honeybees (Apis mellifera) as a function of the probability of reward. Journal of Comparative Psychology, 106, 262-269.

Kehoe, E. J., Horne, A. J., Horne, P. S., \& Macrae, M. (1994). Summation and configuration between and within sensory modalities in classical conditioning of the rabbit. Animal Learning \& Behavior, 22, 19-26.

Klosterhalfen, S., Fischer, W., \& Bitterman, M. E. (1978, September 29). Modification of attention in honeybees. Science, 201, 1241-1243.

LeE, Y., \& Bitterman, M. E. (1990). Learning in honeybees as a function of amount of reward: Control of delay. Animal Learning \& Behavior, 18, 377-386.

Loo, S. K., \& BitTerman, M. E. (1992). Learning in honeybees (Apis mellifera) as a function of sucrose concentration. Journal of Comparative Psychology, 106, 29-36.

Miller, R. R., Barnet, R. C., \& Grahame, N. J. (1995). Assessment of the Rescorla-Wagner model. Psychological Bulletin, 117, 363386.

Pearce, J. M., \& Redhead, E. S. (1993). The influence of an irrelevant stimulus on two discriminations. Journal of Experimental Psychology: Animal Behavior Processes, 19, 180-190.

ResCorla, R. A., \& Coldwell, S. E. (1995). Summation in autoshaping. Animal Learning \& Behavior, 23, 314-326.

ResCORLA, R. A., \& WAGNER, A. R. (1972). A theory of classical conditioning: Variation in the effectiveness of reinforcement and nonreinforcement. In A. H. Black \& W. F. Prokasy (Eds.), Classical conditioning II: Current research and theory (pp. 64-99). New York: Appleton-Century-Crofts.

SMith, B. H., \& COBEY, S. (1994). The olfactory memory of the honeybee Apis mellifera. Journal of Experimental Biology, 195, 91-108.

Sutherland, N. S., \& Mackintosh, N. J. (1971). Mechanisms of animal discrimination learning. New York: Academic Press.

Walker, M. M., Lee, Y., \& BitTerman, M. E. (1990). Transfer along a continuum in the discriminative learning of honeybees (Apis mellifera). Journal of Comparative Psychology, 104, 66-70.

WAGNER, A. R. (1969). Incidental stimuli and discrimination learning. In R. M. Gilbert \& N. S. Sutherland (Eds.), Animal discrimination learning (pp. 83-111). New York: Academic Press.

WEHNER, R. (1981). Spatial vision in arthropods. In H. Autrum (Ed.), Comparative physiology and evolution of vision in invertebrates: C. Invertebrate visual centers and behavior II (pp. 287-616). Berlin: Springer-Verlag.

(Manuscript received September 6, 1994; revision accepted for publication January 17, 1995.) 